\title{
Estrés oxidativo: origen, evolución y consecuencias de la toxicidad del oxígeno
}

\author{
Lucía Constanza Corrales MSc ${ }^{1}$, Maira María Muñoz Ariza ${ }^{2}$ \\ ${ }^{1}$ Docente investigador Universidad Colegio Mayor de Cundinamarca. \\ 2. Estudiantes Universidad Colegio Mayor de Cundinamarca Bogotá, D.C., Colombia.
}

Recibido: 12/07/2012 Aceptado: 02/12/2012

Correspondencia:1corrales@unicolmayor.edu.co

\section{Resumen}

El oxígeno es un elemento que presenta un perfil con doble efecto fisiológico; es esencial para el desarrollo de la vida aerobia y posee efectos tóxicos inherentes a su estructura. Del oxígeno se derivan moléculas inestables denominadas radicales libres que pueden causar daño a nivel celular, cuando se pierde el equilibrio entre dichas moléculas y el sistema de defensa antioxidante que poseen los seres vivos, generando así lo que se denomina estrés oxidativo. Dicho mecanismo posee una estrecha relación con el desarrollo y evolución de una gran variedad de procesos degenerativos, enfermedades y síndromes. Su estudio se ha centrado principalmente en el conocimiento de los mecanismos de su génesis y las formas de; atenuar, disminuir o contrarrestarlas, por medio de los sistemas de defensa antioxidante que posee la célula y la forma de controlar los radicales libres que se generan de forma normal y continua en el organismo resultado de algunos procesos celulares como el relacionado con el metabolismo oxidativo de la mitocondria, proceso necesario para la obtención de ATP.

Palabras clave: estrés oxidativo, radicales libres, especies reactivas de oxígeno, antioxidantes.

\section{Oxidative Stress: origin, evolution and consequences of oxygen toxicity}

\begin{abstract}
Oxygen is an element with dual physiological effect is essential for the development of aerobic life and at the same time can participate in potentially toxic reactions involving oxygen free radicals. Free radicals are unstable molecules that can cause damage at cellular level due an imbalance between free radical production and antioxidant defenses, generating oxidative stress. This mechanism seems to contribute to several pathological conditions including degenerative processes, aging, diseases and syndromes.

Understanding the origin of oxidative stress mechanisms at cellular level, and strategies to reduce it through the cell antioxidant defense system is the focus point of numerous studies. In the same way extensive research has been conducted to find a way to control free radicals production generated continuously through some cellular processes in the body such as oxidative metabolism of the mitochondria and the molecular process required to generate ATP.
\end{abstract}

Keywords: oxidative stress, free radicals, reactive oxygen species, antioxidants. 


\section{Introducción}

En los últimos años ha tomado gran interés el estudio del estrés celular y de los radicales libres en el campo de la medicina, con el fin de conocer a profundidad los mecanismos de autocontrol celular y mejorar la calidad de vida del ser humano (1). Hasta los ańos 70 fue un campo específico de la química, biología y física (2) y hoy en día se ha convertido en una herramienta para conocer la génesis de gran variedad de enfermedades.

Numerosas patologías; gástricas, cardiacas, respiratorias, óseas, multiorgánicas, entre otras son resultado de la evolución de alteraciones morfofisiológicas celulares, debido a la excesiva producción por encima de los niveles fisiológicos normales de moléculas de gran inestabilidad denominadas radicales libres (3). Dicha condición es denominada estrés celular y se caracteriza por presentar un desequilibrio entre la generación y eliminación de especies reactivas (4), situación que se puede derivar de; la disminución de los niveles de las defensas antioxidantes celulares, del aumento de la velocidad de producción de las especies reactivas, o en ciertas ocasiones puede ser resultado de estas dos condiciones (5).

El término especies reactivas hace referencia a dos tipos de moléculas: los radicales libres y los no radicales (6). Este conjunto de moléculas se forma como resultado del metabolismo celular y se encuentran representados dentro de los sistemas biológicos por las especies reactivas de oxígeno (ROS) y por las especies reactivas de nitrógeno (RNS)(7), que se originan tanto en procesos fisiológicos normales como en procesos patológi$\cos (8)$. Sin excluir que también existen especies reactivas procedentes de otros elementos como cloro (RClS) y Bromo (RBrS)(9), aunque ROS y RNS son los dos grandes grupos implicados dentro de la biología redox $(9,10)$.

\section{Radicales libres (RL)}

Los radicales libres son moléculas capaces de existir de forma independiente conteniendo en su última orbita uno o más electrones desapareados, los cuales le otorgan a estas carácter paramagnético(11) que las torna muy inestables y altamente reactivas con capacidad para combinarse inespecíficamente, con las diferentes moléculas que integran la estructura celular y los derivados de estas, y con la capacidad de atacar cualquier tipo de biomolécula $(12,13)$. Por su configuración electrónica estos radicales presentan una vida media corta. Sin embargo, en diversos estudios, se ha demostrado que la reactividad de estas moléculas se correlaciona inversamente con su vida media y capacidad de difusión en el medio celular(14).

Los radicales libres se pueden formar a partir de diversos mecanismos, siendo la adición de un electrón a una molécula estable el más común (14). Una vez que estos son formados, buscan el modo de conseguir una configuración electrónica estable, razón por la cual interactúan con otras moléculas a través de reacciones de óxido reducción (redox). En dichas circunstancias, hay una transferencia de electrones que necesariamente implican la reducción (ganancia de electrones) y oxidación (pérdida de electrones) de las moléculas participantes. Dicho mecanismo genera que la producción de radicales libres sea una reacción en cadena, ya que al reaccionar un radical libre con una molécula no radical inevitablemente esta última pasa a ser un RL. Dicha reacción en cadena solamente se detendrá cuando dos radicales libres se encuentren y reaccionen entre sí $(15,16)$.

Estas reacciones bioquímicas se encuentran clasificadas en tres grupos:

Reacciones de iniciación. Es la formación de un radical libre a partir de no radicales.

$$
\mathrm{AB}+\mathrm{C} \rightarrow \mathrm{A} \bullet+\mathrm{D}+\mathrm{E}
$$

Reacciones de propagación. Consisten en la formación de un radical libre cuando reacciona una molécula estable con un radical libre.

$$
\mathrm{A} \bullet+\mathrm{CD} \rightarrow \mathrm{AC}+\mathrm{D} \bullet
$$


Reacciones de terminación. Hace referencia a la reacción química entre dos radicales libres, en donde sus electrones desapareados son cancelados y se genera un producto estable(17).

$$
\mathrm{A} \bullet+\mathrm{D} \bullet \rightarrow \mathrm{AD}
$$

Clasificación de los RL. Algunos autores dentro de sus investigaciones han llegado a clasificar a los RL de acuerdo al grupo funcional presente en la molécula; bromo, nitrógeno, tioles, fósforo, oxígeno, cloro, entre otros, aunque los radicales libres de oxígeno reactivo son los más comunes y de mayor relevancia debido a su participación en los diferentes procesos aeróbicos (18-20).

Oxígeno. Es un elemento químico de gran abundancia sobre la tierra. Se encuentra en un $53.8 \%$ de la corteza terrestre y su forma estructural $\mathrm{O}_{2}$ hace parte de cerca del $21 \%$ de la composición del aire (21). Dentro del geosistema de la tierra, el oxígeno se encuentra en combinación con otros elementos. En la litosfera está combinado con silicio, en la arena; en la hidrosfera con hidrógeno, formando agua: océanos, ríos, lagos y nacimientos de agua; y en la atmósfera se encuentra de forma gaseosa (22).

Dentro del marco histórico que envuelve a este elemento, su descubrimiento continúa siendo un evento polémico porque tres personajes son los protagonistas de este acontecimiento; en primera instancia, muchos atribuyen el descubrimiento a Carl Wilhelm Scheele un farmacéutico y químico sueco que consiguió producir oxígeno y relató su descubrimiento, en sus trabajos realizados entre los años de 1770 a 1773 y recopilados en su libro Chemische *Abhandlug Von Der Luft Und Dem Fever (Tratado químico del agua y del fuego), que se publicó en 1777, y en el que se describen ciertas características del oxígeno (23-25).

De igual forma, numerosas publicaciones indican que hacia el año de 1774, el químico inglés Joseph Prestley obtuvo este elemento y realizó la publicación de este hallazgo antes que Scheele, razón por la cual Prestley es reconocido dentro del campo científico como el verdadero descubridor del oxígeno (24). Así mismo, el químico francés Lavoisier produjo oxígeno, a través del calentamiento de unos trozos de metal entre los ańos de $1774 \mathrm{y}$ 1777. Sin embargo, él ya conocía con antelación de las investigaciones realizadas por Scheele (25).

Con relación al origen del oxígeno es importante recordar que en un comienzo la atmósfera era anóxica, se encontraba formada por vapor de agua $\left(\mathrm{H}_{2} \mathrm{O}\right)$, metano $\left(\mathrm{CH}_{4}\right)$, sulfuro de hidrógeno $\left(\mathrm{H}_{2} \mathrm{~S}\right)$, dióxido de carbono $\left(\mathrm{CO}_{2}\right)$, amoniaco $\left(\mathrm{NH}_{3}\right)$, monóxido de carbono (CO) e hidrógeno $(\mathrm{H})$. Los organismos vivos, en aquel entonces, realizaban la fermentación de materiales orgánicos para obtener energía a partir de moléculas de $\mathrm{H}_{2} \mathrm{O}$, de $\mathrm{CO}_{2}$ y de $\mathrm{NH}_{3}$, con la participación de varias fuentes energéticas como la radiación ultravioleta, las tormentas eléctricas y la actividad volcánica (21).

Posteriormente, aparecieron las bacterias fotosintéticas. Sin embargo, estas no liberaban oxígeno como subproducto. Los primeros organismos en poder realizar fotosíntesis aeróbica fueron los predecesores de las algas azul verdosas, responsables de la aparición de este gas libre en la atmósfera hace aproximadamente dos mil millones de años. De esta forma, para el momento de la aparición de las células eucariotas el oxígeno existía hacía tiempo (21).

Sin duda alguna el oxígeno es un elemento imprescindible para la vida; es usado por los organismos aeróbicos para aumentar la extracción de energía proveniente de los nutrientes, mediante su oxidación y liberación en forma de Adenosín trifosfato (ATP) (26).

Por otro lado, a través de diferentes estudios se ha encontrado que el oxígeno puede llegar a ser tóxico, cuando los seres vivos se exponen a altas concentraciones por encima de los niveles atmosféricos, lo que puede conllevar a dańos tanto reversibles como irreversibles, llegando hasta la muerte celular (27). 
Dicho efecto nocivo involucra el proceso de reducción parcial del oxígeno, mediante el que se obtienen las especies reactivas de oxígeno (EROS). Esta moléculas pueden convertirse en peligrosos productos del metabolismo celular, debido a que cuando se rompe el equilibrio entre sustancias prooxidantes (EROS) y sustancias antioxidantes se genera estrés oxidativo y por ende diversos procesos patológicos (28), Figura 1.

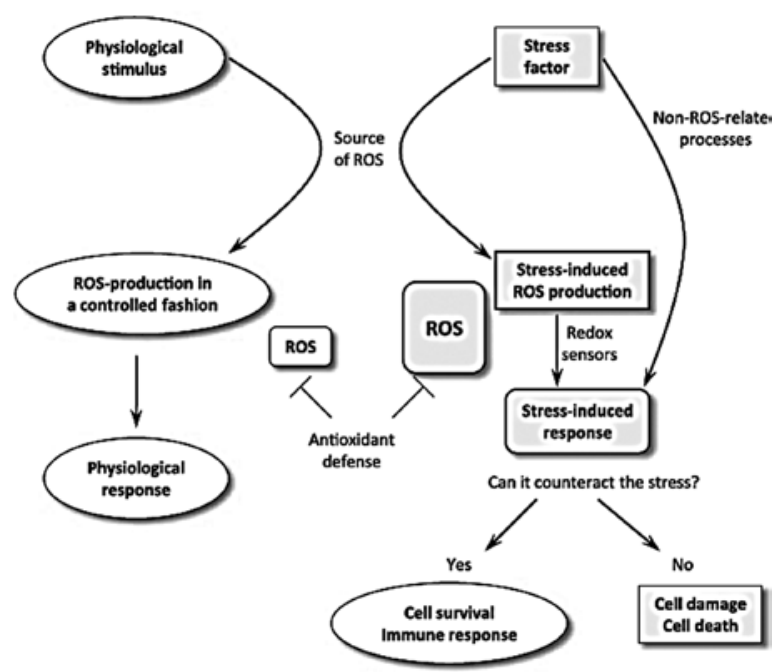

Figura 1. Respuesta celular frente a la formación de EROS a través de estímulos fisiológicos(28). Fuente: Review Reactive Oxygen an nitrogen species in normal physiological processes, República Checa, Pourova J. 2010:22.

Especies activas de oxigeno. En su estado natural, el oxígeno es un birradical; lo que indica que posee dos electrones desapareados en los orbitales p antiligantes, con el mismo número cuántico de espín (29), que evita que reaccione como un radical libre y le confiere la característica de ser un elemento altamente oxidante. Es decir, que le permite aceptar electrones de otras moléculas $(30,31)$.

De igual forma, el oxígeno solamente puede reaccionar con moléculas que posean una configuración electrónica semejante a la suya. Razón por la cual queda impedido (por restricción de spin) para reaccionar con la mayoría de moléculas existentes, ya que estas no son birradicales, evitando de esta manera que se lesionen ciertos blancos celulares importantes. Así mismo, en los procesos de transferencia de electrones o de absorción de energía se pueden generar las especies reactivas de oxígeno (32) que involucran, tanto a los radicales libres de oxígeno como a las moléculas no radicales derivadas de este mismo elemento, que aunque no son RL poseen la capacidad de generar RL, Tabla 1.

Cuando el oxígeno oxida un compuesto este se reducirá gracias a la ganancia de electrones (un electrón por vez), es decir, al adicionar un electrón al oxígeno cuando este se encuentra en estado fundamental se forma el anión superóxido $\left(\mathrm{O}_{2}{ }^{-}\right)$, si se añade otro electrón como producto se obtendrá el anión peróxido, el cual no es un radical libre ya que no posee electrones desapareados (33).

\begin{tabular}{|c|c|c|}
\hline \multicolumn{3}{|c|}{$\begin{array}{l}\text { Cuadro1 } \\
\text { CARACTERÍSTICASDELASPRINCIPALESESPECIESREACTIVASDELOXÍGENO(EROS) } \\
\text { CHARACTERISTICSOFTHEMAINREACTIVEOXYGENSPECIES(ROS) }\end{array}$} \\
\hline ROS & Symbol & Characteristics \\
\hline Superoxide & $\mathrm{O}_{2}$ & $\begin{array}{l}\text { Intermediatein } \mathrm{O}_{2} \text { reductionto } \mathrm{H}_{2} \mathrm{O} \text {.Goodreductantandbadoxidant.Itis } \\
\text { important becauseitgenerates more } \mathrm{ROS} \text {, suchas } \mathrm{OH} \cdot \mathrm{AndH}_{2} \mathrm{O}_{2}\end{array}$ \\
\hline Hydroxyl & HO & $\begin{array}{l}\text { Themostpowerful oxidantinbiological systems.Itisgenerated fromFenton } \\
\text { andHaber-Weissreactions. }\end{array}$ \\
\hline Peroxyl & $\mathrm{ROO}$ & Low oxidantability, buthigh diffusibility. \\
\hline Alkoxyl & Ro- & Mediumoxidantabilitywithlipids. \\
\hline Hydrogen peroxide & $\mathrm{H}_{2} \mathrm{O}_{2}$ & $\begin{array}{l}\text { Originated from } \mathrm{O}_{2}=\text { dismutation by the SODenzyme. Canoriginatevery } \\
\text { reactive FR when itreacts withtransitionmetals. }\end{array}$ \\
\hline Hypochlorousacid & HCO & Formed throughmieloperoxidaseaction, it is presentinneutrophils on $\mathrm{H}_{2} \mathrm{O}_{2}$ \\
\hline Singlet oxygen & ${ }^{1} \mathrm{O}_{2}$ & Molecularlyexcited oxygen throughsunlightandradiation.Highly oxidant. \\
\hline
\end{tabular}

Tabla 1. Especies reactivas de oxígeno (34).

Fuente: Review Cellular defenses against damage from reactive oxygen species. Yu P. 1994. 
De la misma forma, cuando se da la reducción parcial del oxígeno por aceptación de dos electrones se da paso a la formación de peróxido de hidrógeno $\left(\mathrm{H}_{2} \mathrm{O}_{2}\right)$. Sin embargo, la unión que se da entre O-O en el peróxido de hidrógeno es muy débil y se puede llegar a descomponer dando así origen al radical hidroxilo $(\mathrm{HO})$, el cual posee una alta reactividad. Sin embargo, cuando el oxígeno acepta cuatro electrones se forma una molécula de agua $\left(\mathrm{H}_{2} \mathrm{O}\right)(33)$, Figura 2.

El oxígeno en el proceso de reducción parcial también puede absorber energía de nuevo y pasar a un estado excitado de mayor reactividad y energía, denominado oxígeno singlete(21).

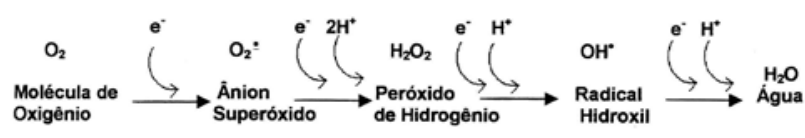

Figura 2. Esquema de producción de las especies activas de oxígeno (35).

Fuente: R. A toxicidade do oxigênio. Meneghini. 1987.

Por otra parte, los radicales libres son formados a través de diferentes procesos metabólicos aeróbicos esenciales del organismo (36), razón por la cual, son considerados especies químicas naturales, ya que de modo continuo son generadas por los seres vivos(37) y participan en varios procesos fisiológicos del organismo como; producción de energía, regulación del crecimiento celular, síntesis de sustancias biológicas (colágeno y prostaglandinas), defensa de tipo inmune (fagocitosis), señalización intracelular y favorecimiento en la quimiotaxis (38-40).

No obstante, las ERO por su alta reactividad pueden llegar a causar diferentes daños a nivel celular, tales como; oxidación de lípidos (lipoperoxidación), de grupos sulfidril de las proteínas, de bases púricas y pirimidínicas (41), generando alteraciones celulares que pueden ser el primer paso para el desarrollo de patologías. Diversos estudios han comprobado que las ERO están implicadas en enfermedades como VIH, Parkinson, artritis reumatoidea, cirrosis, entre otras (42).

\section{Principales espécies reactivas}

Anion superóxido $\left(\mathrm{O}_{2}^{-}\right)$Es el radical más abundante y común a nivel celular. Se forma principalmente en la cadena de transporte de electrones y en la fagocitosis para ser usado como defensa bactericida $(43,44)$. Es generado en reacciones de autoxidación y enzimáticas en diferentes organelos celulares:

Mitocondrias. Por la oxidación de la semiquinona de la ubiquinona $\left(\mathrm{UqH}^{\circ}\right)$

$$
\mathrm{O}_{2}+\mathrm{UqH}^{\cdot} \quad \mathrm{O}_{2}^{-}+\mathrm{Uq}^{-}+\mathrm{H}^{+}
$$

- Por la oxidación de la semiquinona de la flavina de la NADH deshidrogenasa $\left(\mathrm{FpH}^{\circ}\right)(45,46)$.

$$
\mathrm{O}_{2}+\mathrm{FpH}^{\cdot} \quad \mathrm{O}_{2}^{-}+\mathrm{Fp}+\mathrm{H}^{+}
$$

Retículo endoplásmico. A través del citocromo $\mathrm{P}_{450}$ y de la flavoporteína NADPH - citocromo $\mathrm{P}_{450}$ reductasa(21):

$$
\mathrm{O}_{2}+\mathrm{Fe}^{2+} \quad \mathrm{O}_{2}+\mathrm{Fe}^{3+}
$$

Citosol. Por acción de la enzima xantina oxidasa (1):

$2 \mathrm{O}_{2}+$ xantina $\mathrm{O}_{2}+$ ácido úrico

Dentro de las sustancias que se oxidan y permiten la generación de esta forma reactiva se encuentran; la hemoglobina, mioglobina y catecolaminas. De igual forma, enzimas como xantina oxidasa y NADPH oxidasa generan radicales superóxido. También, dentro de la fagocitosis es producido superóxido gracias a la activación de la enzima NADPH oxidasa que se encuentra en la membrana celular (32).

Peróxido de hidrógeno $\left(\mathrm{H}_{2} \mathrm{O}_{2}\right)$ No es un radical libre, pero es una forma reactiva de gran importancia, ya que posee la capacidad de generar el radical hidroxilo cuando se encuentra en presencia de metales como el hierro. Esta ERO, es formada prin- 
cipalmente en la matriz mitocondrial, al realizar la reducción parcial de oxígeno o en la dismutación del radical superóxido por la enzima superóxido dismutasa (SOD)(47). Aunque también se genera en otros organelos celulares como el retículo endoplásmico, peroxisomas y citosol, a través de las siguientes reacciones:

Mitocondria. A partir de la dismutación del $\mathrm{O}_{2}^{-}$, catalizada por la superóxido dismutasa.

$$
2 \mathrm{O}_{2}^{-}+2 \mathrm{H}^{+} \quad \mathrm{H}_{2} \mathrm{O}_{2}+\mathrm{O}_{2}
$$

Retículo endoplásmico. Por la autooxidación del citocromo $\mathrm{P}_{450}\left(\mathrm{FMNH}_{2}\right)$ de la citocromo c NADPH reductasa (21):

$$
\mathrm{FMNH}_{2}+\mathrm{O} 2 \quad \mathrm{H}_{2} \mathrm{O}_{2}+\mathrm{FMN}
$$

Peroxisomas. En este organelo se encuentran diferentes proteínas que pueden dar origen al $\mathrm{H}_{2} \mathrm{O}_{2}$ como lo son: la urato oxidasa, la 1- $\alpha$-hidroxiácido oxidasa y la D-amnoácido oxidasa (21). Además, algunos microorganismos como bacterias y micoplasmas liberan peróxido de hidrógeno, el cual por su capacidad de atravesar membranas biológicas puede causar daño en el huésped a nivel celu$\operatorname{lar}(32)$.

Radical Hidroxilo $\left(\mathrm{OH}^{\circ}\right)$ Posee una alta reactivi$\mathrm{dad}$, esto es inversamente proporcional a su vida media, lo que indica que tiene una vida media bastante corta, debido a que reacciona de manera rápida e inespecífica con los blancos celulares más cercanos (DNA, proteínas, lípidos y carbohidratos) (48). Tiene una capacidad superior a las demás ERO de causar daño a nivel celular, debido a que las células no cuentan con un sistema enzimático antioxidante contra este radical (12).

La formación de este radical a nivel celular se da por varios mecanismos, dentro de los cuales se encuentra; la radiolisis del agua que contiene la célula cuando esta es expuesta a radiaciones ionizantes como los rayos $\mathrm{X}$ y los rayos gamma. Además, al reaccionar el radical superóxido con el peróxido de hidrógeno se produce el radical hidroxilo a través de la reacción Haber - Weiss (12), Figura 3.

$$
\mathrm{O}_{2}^{\cdot-}+\mathrm{H}_{2} \mathrm{O}_{2} \quad \mathrm{OH}^{\bullet}+\mathrm{OH}^{-}+\mathrm{O}_{2}
$$

Figura 3. Producción de radical hidroxilo a través de la reacción de Haber- Weiss.

Por otro lado, la formación del radical hidroxilo, desde el peróxido de hidrógeno, puede llegar a ser catalizada por la presencia de iones de metales de transición como lo muestra la reacción de Fenton (12), Figura 4.

$$
\mathrm{Fe}^{2+}+\mathrm{H}_{2} \mathrm{O}_{2} \longrightarrow \mathrm{Fe}^{3+}+\mathrm{OH}^{\cdot}+\mathrm{OH}^{-}
$$

Figura 4. actividad catalítica del hierro en la producción de radical hidroxilo.

Existen elementos que promueven la formación de radicales libres como son los iones de metales de transición, ya que a medida que cambian de estado de valencia van perdiendo o ganando electrones, en el organismo los más abundantes son el hierro y el cobre (49). Aunque los iones hierro que se encuentran en la transferrina y lactoferrina en presencia de $\mathrm{pH}$ fisiológico no participan de la producción de este RL, el hierro que se encuentra en la ferritina pueda ser mobilizado por moléculas reductoras como el superóxido o el ascorbato y catalizar la formación del radical hidroxilo (50).

Oxígeno Singlete $\left(\mathrm{O}_{2}{ }^{1}\right)$ La especie reactiva oxígeno singlete es producida en diferentes procesos, como; la fagocitosis, inducción luminosa, reacciones catalizadas por peroxidasas, entre otras $(51,52)$. Es capaz de modificar diferentes biomoléculas como el DNA y causar daños en las proteínas a través de la oxidación de ciertos grupos esenciales de aminoácidos entre los cuales se encuentran; triptófano, metionina, histidina y resíduos de cisteína. De igual forma, da inicio a la lipoperoxidación generando radicales como el alcoxilo $(\mathrm{RO} \bullet$ ) y peroxilo $(\mathrm{ROO} \bullet)(12,53)$. 
Posee una vida media corta de aproximadamente 0.5 microsegundos. Se caracteriza por que en su estado molecular no tiene restricción en la transferencia de electrones, por tal motivo es altamente reactivo $(51,52,54)$.

\section{Fuentes de radicales libres}

\section{Fuentes endógenas}

Mitocondría. El metabolismo oxidativo se da en la mitocondria, conocida como el organelo con mayor importancia dentro de la producción de ATP, molécula indispensable para el organismo porque de ella es liberada la energía necesaria para los diferentes procesos vitales(55).

La mitocondria es considerada como la principal fuente de radicales libres, ya que al producirse el metabolismo oxidativo se da la reducción tetraelectrónica del oxígeno con un consumo del 95 al 98\% de este elemento. El restante 2 - 5\% de oxígeno tiene una tendencia de recibir un electrón por vez, reducción monoelectrónica, formando durante estas reacciones una serie de intermediarios tóxicos: especies reactivas de oxígeno $(55,56)$, Figura 5.

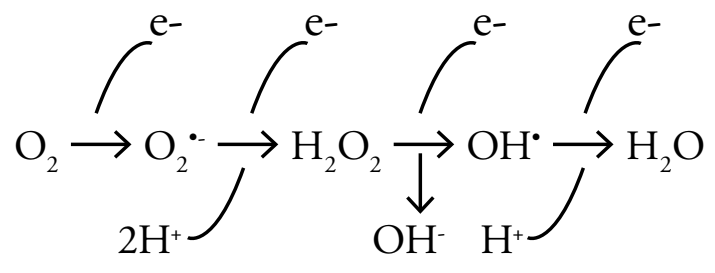

Figura 5. Generación de ERO a partir de la reducción de oxigeno (49).

Cada mitocondria produce alrededor de $10-7 \mathrm{M}$ $\mathrm{RL} /$ día. Sin embargo, alteraciones dentro de la cadena de transporte de electrones pueden aumentar estos niveles en la mitocondria (56). Diferentes investigadores, como Nicholls y Ferguson, han sugerido que dentro de este organelo el radical O2 - se forma dentro de los complejos I y III de la cadena respiratoria. En medio de este proceso de transferencia de electrones la coenzima Q es oxidada formando la ubisemiquinona, la cual es un radical intermediario que al entrar en contacto con el oxígeno produce radicales superóxido (45), Figura 6.

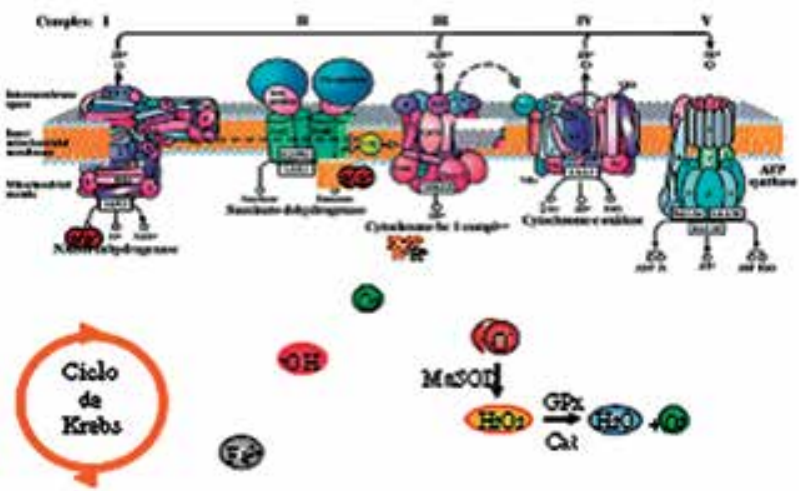

Figura 6. Formación de EROS vía complejos de cadena respiratoria(46).

Fuente: Mitochondrial DNA repair and aging. Mandavilli B, Van Houten B. 2002.

No obstante, estudios han descubierto que la mitocondria también es capaz de producir otras especies reactivas que tienen significado biológico y que se forman como consecuencia de una reacción en cascada (55); en la cual se produce óxido nítrico (NO*), a través de la acción de la óxido nítrico sintasa (NOS) calcio dependiente, que se encuentra localizada en la membrana mitocondrial (57).

Dicho RL puede tener un rol fisiológico, a través de la modulación de la velocidad del flujo de electrones en condiciones fisiológicas como la hipoxia. Así mismo, se puede combinar con $\mathrm{O}_{2}{ }^{--}$y dar origen al peroxinitrito (ONOO${ }^{-}$) encargado de reaccionar directamente con las hemoproteínas inhibiendo la capacidad de transportar electrones, mediante la inactivación de los complejos I y III de la cadena respiratoria $(58,59)$. Por esto, se produce una acumulación de electrones en la cadena respiratoria lo que propicia, que en ciertos componentes mitocondriales, se aumenten los niveles de producción de $\mathrm{O}_{2}{ }^{-}$al igual que de $\mathrm{ONOO}^{-}$y de $\mathrm{H}_{2} \mathrm{O}_{2}$, lo que conlleva a la oxidación de proteínas y liberación de Citocromo $\mathrm{C}$ al citoplasma, dando así inicio a la apoptosis celular (60). 
Peroxisomas. Estos organelos poseen una gran cantidad de enzimas que producen $\mathrm{H}_{2} \mathrm{O}_{2}$ y también actúan en el metabolismo del ácido glioxílico, de bases purinicas y de aminoácidos (29). Estos organelos celulares al igual que los gliosomas (organelos encargados de hacer el ciclo del glioxilato) poseen altas concentraciones de catalasa, que convierten al $\mathrm{H}_{2} \mathrm{O}_{2}$ en $\mathrm{H}_{2} \mathrm{O}$ y $\mathrm{O}_{2}$, aunque en aquellos casos en donde el peróxido de hidrógeno no es dismutado por el sistema peroxisomal se produce inevitablemente daño celular $(49,61)$.

Citocromo P-450. Se encuentra en el retículo endoplasmático y es un lugar importante de producción de RL. Su función consiste en catalizar las reacciones que generan $\mathrm{O}_{2}{ }^{-}$, mediante mecanismos dependientes de NADPH. Este sistema que contiene citocromos presenta las condiciones adecuadas para que se generen RL, debido a que cuentan con la presencia de iones de metal de transición, oxígeno y además se realiza la transferencia de electrones (49).

Las enzimas que contiene este complejo son responsables del metabolismo oxidativo de los xenobióticos,sustratos exógenos, en especial de los compuestos lipofílicos, entre los cuales los de mayor relevancia son; los fármacos terapeúticos, productos químicos y subproductos industriales (62).

De igual forma, previenen efectos de toxicidad de drogas tales como; el benzeno, alcohol, insecticidas clorados, paraquat, antimaláricos, antitumorales y antifúngicos (63). Sin embargo, algunos compuestos que sufren esta detoxificación por reacciones catalizadas en el citocromo P-450 se convierten en intermediarios reactivos que inician la peroxidación lipídica, ocasionando daño en el DNA y en la membrana microsomal (64).

Fagocitosis. Durante la fagocitosis se producen ERO benéficas, debido a que estas son la primera línea de defensa contra los patógenos(7). Al activarse en las células fagocíticas (leucocitos polimorfonucleares) a través de; los mediadores proinflamatorios, o de la presencia de productos bacterianos, víricos o de parásitos, se da una "explosión" oxidativa en la que se produce gran cantidad de radicales $\mathrm{O}_{2}{ }^{--}, \mathrm{H}_{2} \mathrm{O}_{2}, \mathrm{OH}^{\bullet}$ y $\mathrm{NO}$ para destruir las células infectadas (65).

Los polimorfonucleares, poseen en sus membranas la enzima NADPH oxidasa, que genera $\mathrm{O}_{2}{ }^{-}$ , el cual en presencia de iones hierro pasa a ser $\mathrm{OH}^{*}$ o puede ser dismutado por la SOD, y así generar $\mathrm{H}_{2} \mathrm{O}_{2}(12)$.

Xantina deshidrogenasa. Se encuentra predominando en los endotelios y depura a las xantinas. No obstante, al combinarse la actividad de esta enzima con la de la xantina oxidasa, se generan $\mathrm{O}_{2} \cdot{ }^{-}$y $\mathrm{H}_{2} \mathrm{O}_{2}(12)$.

\section{Fuentes exógenas}

No obstante, los RL no solamente son formados de manera endógena. Existen otros factores o fuentes que favorecen la formación de los radicales libres, tales como; la exposición a rayos $\mathrm{X}$, al ozono, al tabaco, a contaminantes del aire y productos químicos industriales, además de ciertos medicamentos $(36,65)$, que promueven el aumento de producción de ERO, Tabla 2. 


\begin{tabular}{|c|c|}
\hline Contaminantes & $\begin{array}{l}\text { Fibras de asbestos } \\
\text { Polvo de minerales } \\
\text { Ozono } \\
\text { Monóxido de carbono } \\
\text { Óxido nitrico y Dióxido de nitrógeno } \\
\text { Silice } \\
\text { Solventes } \\
\text { Toxinas } \\
\text { Hipocloritos } \\
\text { Dióxido de sulfuro } \\
\text { Bifenilos policlorados } \\
\text { Paraquat y Diquat }\end{array}$ \\
\hline Drogas & $\begin{array}{l}\text { Acetaminoceno } \\
\text { Coprofloxacino } \\
\text { Antidepresivos tricíclicos } \\
\text { Nitrofurantoínas } \\
\text { Antidiabéticos } \\
\text { Bleomicina } \\
\text { Doxorubicina }\end{array}$ \\
\hline lones metálicos & $\begin{array}{l}\text { Hierro } \\
\text { Cobre } \\
\text { Cadmio } \\
\text { Niquel } \\
\text { Cromo } \\
\text { Mercurio }\end{array}$ \\
\hline Radiaciones & $\begin{array}{l}\text { Ultravioleta } \\
\text { Rayos X } \\
\text { Gamma }\end{array}$ \\
\hline Dieta & $\begin{array}{l}\text { Ácidos grasos poliinsaturados } \\
\text { Glucosa }\end{array}$ \\
\hline Otros & $\begin{array}{l}\text { Tabaco } \\
\text { Ejercicio intenso }\end{array}$ \\
\hline
\end{tabular}

Tabla 2. Principales factores externes que incrementan la producción de ERO (65).

Fuente: Caracterización de derivados polifenólicos obtenidos de fuentes naturales. Citotoxicidad y capacidad antioxidante frente a estrés oxidativo en modelos celulares. Ugartondo V. 2009

Sistema de defensa antioxidante. Los organismos evolutivamente desarrollaron sistemas de defensa antioxidante, para poder neutralizar a las especies reactivas de cualquier elemento (12).

Un antioxidante es cualquier sustancia que retarda o previene la oxidación de un sustrato oxidable, que puede ser; lípido, proteína, DNA, o cualquier otro tipo de molécula (66).

El sistema de defensa antioxidante se encuentra formado por elementos tanto enzimáticos como no enzimáticos, que actúan conjuntamente para así proteger a la célula. El componente de tipo enzimático se considera como la primera línea de defensa primaria, y se encarga de evitar el acúmulo de EROS como; el anión superóxido y el peróxido de hidrógeno, catalizando la transferencia de electrones de un sustrato hacia los RL $(34,67)$.

Dentro del sistema de defensa antioxidante enzimático se encuentran las siguientes enzimas:

Superóxido Dismutasa (SOD). Esta enzima posee como función catalizar la dismutación del $\mathrm{RL} \mathrm{O}_{2}{ }^{*}$ a $\mathrm{H}_{2} \mathrm{O}_{2}$, el cual es menos reactivo y puede llegar a ser degradado por otras enzimas como la catalasa o la glutationa peroxidasa $(68,69)$. Esta enzima, se encuentra en cuatro isoformas:

1. Sod1 o SodCuZn: homodímero de cobre y zinc que se encuentra en el espacio citoplasmático intracelular(47).

2. Sod2 o Sodmn: tetrámero que contiene manganeso en su sitio activo y se encuentra en la matriz mitocondrial(47).

3. Sod3 o ECSod: es la más recientemente caracterizada. Es un tetrámero que contiene cobre y zinc y posee un péptido señalizador que la direcciona hacia el espacio extracelular. Sin embargo, su papel en los diferentes estados fisiológicos, hasta ahora no está del todo claro (70).

4. SodFe: contiene hierro y se encuentra en bacterias, algas y vegetales (12).

Catalasa. Es una hemoproteína que se concentra principalmente en los peroxisomas y en las mitocondrias, se encarga de catalizar la descomposición del peróxido de hidrógeno en agua y oxígeno (34).

Glutatión peroxidasa (GPx). Es una selenoproteína, en presencia de GSH, como agente reductor, cataliza la reducción del peróxido de hidrógeno y otros hidroperóxidos orgánicos en agua y alcohol, respectivamente (71). Esta enzima posee tres isoformas: 
1. GSH - Px celular: o clásica, se encuentra en todas las células y reduce el peróxido de hidrógeno e hidroperóxidos orgánicos libres y los convierte como se mencionó anteriormente en agua y alcohol $(72,73)$.

2. GSH - Px plasmática o extracelular: es caracterizada a partir de plasma humano y se sintetiza en las células proximales al riñón $(72,73)$.

3. GSH - Px fosfolípido hidroperóxido: su función es proteger contra la lipoperoxidación mediante la reducción de ácidos grasos en las membranas celulares, previene también la oxidación de las lipoproteínas de baja densidad $(72,73)$.

4. GSH- Px gastrointestinal: reduce los hidroperóxidos de colesterol y protege contra la toxicidad generada por la ingestión de hidroperóxidos lipídicos (74).

Los antioxidantes de tipo no enzimáticos son un conjunto de moléculas tanto hidrófobas como hidrofílicas que tienen como función; capturar RL y generar moléculas menos nocivas para la célula, mediante la adición de un electrón al RL con el objetivo de estabilizarlo $(68,75)$.

Dentro del sistema de defensa antioxidante no enzimático se encuentra:

Vitamina $C$. Se encuentra a nivel intra y extracelular en la forma de ascorbato, actúa directamente sobre los radicales superóxido, hidroxilo y algunos hidroperóxidos lipídicos, además de actuar sobre el tocoferoxilo, transformándolo a vitamina E. Sin embargo, el ascorbato puede llegar a ser un potente prooxidante cuando se encuentran presentes excesivas concentraciones de iones hierro y cobre (68).

Glutatión. Consiste en un tripéptido que presenta una distribución tisular variable y es considerado el compuesto tiólico de bajo peso molecular, de mayor abundancia en las células de los mamíferos. Actúa sobre el peróxido de hidrógeno, superóxido y el radical hidroxilo (71).
Flavonoidespolifenólicos. Dentro de los flavonoidespolifenólicos se encuentran un amplio grupo de compuestos fenólicos (catecinas, cianidinas, quercetinas) que actúan como quelantes de metales y que además, capturan de forma in vitro ERO y ERN. Pueden ser de tipo lipo e hidrosolubles y se ubican tanto intra como extracelularmente (75). Dentro de este grupo se encuentran la vitamina E, la vitamina $A$, la ubiquinona (coenzima $Q$ ), la albúmina, el ácido lipoico, el fibrinógeno, la bilirrubina y la glucosa $(18,71,76)$.

\section{Implicaciones del estrés oxidativo}

Mantener el control de los niveles de las especies reactivas, permite que a condiciones bajas y moderadas estas moléculas sean beneficiosas dentro de los procesos celulares. Pese a esto, cuando los niveles de las especies reactivas se elevan pueden generar estrés oxidativo (aumento de ERO), que trae como consecuencia daños celulares irreparables (77).

Las ERO, han sido implicadas dentro de numerosas patologías como lo son:

Corazón: trombosis, hipertrofia; sistema nerviosos central: parkinson y demencia; articulaciones: artritis reumatoide; tracto gastrointestinal: pancreatitis y hepatotoxicidad; ojos: cataratas y retinopatía; multiorgánicas: inflamación, intoxicaciones, envejecimiento, isquemia, cáncer; vasos: ateroesclerosis; eritrocitos: anemia de fanconi y malaria; pulmón: asma; piel: soriasis y quemaduras (78).

En todas estas enfermedades las alteraciones celulares incluyen reclutamiento de macrófagos, daño mitocondrial, interferencia con defensas antioxidantes, aumento del calcio intracelular y conversión de la xantina deshidrogenasa a xantina oxidasa, lo cual puede llevar al organismo a alguna de las siguientes tres posibilidades:

1. Adaptación: ya que hay aumento de la actividad de los sistemas de defensa antioxidante, que protege a la célula frente a daños futuros. 
2. Daño en tejidos: por daño en lípidos, proteínas y carbohidratos.

3. Muerte celular: por necrosis o apoptosis (78).

\section{Métodos de evaluación del estrés oxidativo}

Un gran problema dentro del estudio de los RL y del estrés oxidativo han sido las mediciones in vivo de los RL, debido a que estos poseen una vida media bastante corta. Sin embargo, la única técnica que permite la medición de RL de forma directa es la electron spin resonance (ESR) (49). Por esto, se han implementado otras técnicas que permiten ver el dańo causado por los RL y la actividad de ciertos parámetros dentro del estrés oxidativo como son; evaluación de proteínas (técnica de Lowry y método de Bradford), quimioluminiscencia, sustancias reactivas al ácido tiobarbitúrico (TBARS), actividad de SOD, determinación de catalasa, determinación de la enzima Glutation peroxidasa, medición de Glutation S- Transferasa, medición de glutatión y determinación de nitritos y nitratos (31).

Finalmente, el estrés oxidativo y los radicales libres son actualmente tema relevante de investigación y atención en el campo científico. A través de su estudio se han comprendido los diferentes fenómenos generados a nivel celular en el desarrollo de diferentes patologías. De igual forma, el estudio exhaustivo de los mecanismos de producción de estrés oxidativo, a través de diferentes métodos de laboratorio y de experimentación animal, han sido la base para comenzar a diseñar estrategias terapéuticas que ayuden a mantener y recuperar el equilibrio entre sustancias oxidantes y prooxidantes, para así evitar el progreso y desarrollo de patologías asociadas a este proceso.

\section{Referencias bibliográficas}

1. Possa N. Estresse Oxidativo e Antioxidantes. Primera edición. Canoas- RS (Brasil). Editora da ULBRA. 2002.

2. García J, García B, Morin M, et al. Radicales libres: impacto médico. México; 1993; 13 (3): 75 - 83.

3. Bello a. Dano oxidativo e regulação Biológica pelos Radicais LivresCanoas- RS (Brasil). Editora ULBRA. 2002.

4. Clayton C, Barbosa A, Pontes P, et al. Oxidative Stress and Epilepsy: Literature Review. Oxidative Medicine and Celllular Longevity; 2012: 1 -12.

5. Pavanatto M, Llesuy S. Espécies ativas de oxigênio e de nitrogênio. Canoas- RS (Brasil). Editora da ULBRA. 2008: 13 -24.

6. Lozada S, García L. Estrés oxidative y antioxidants: cómo mantener el equilibrio. Rev Asociación Colombiana de Dermatología. 2009; 17: 172 - 179.

7. Nogueira E. Estudio de la respuesta celular a SOK1 y mecanismos de regulación. [Tesis doctoral]. Universidad de Santiago de Compostela. Departamento de Fisioloxía. 2008

8. Moura G. Efeito dos principais metabolitos acumulados na academia isovalérica sobre parámetros de estresse oxidativo em cerebro de ratos. [Tesis de maestría]. Canoas- RS (Brasil); 2007.

9. Halliwell B. Reactive species and antioxidants redox biology is a fundamental theme of aerobic life. Plant Physiol 2006; 141: $312-22$.

10. Kohen R, Nyska A. Oxidation of biological systems: oxidative stress pehnomena, antioxidants, redox reactions, and methods for their quantification. Toxicology and Pathology; 2002. 31: $620-650$.

11. Pryor W. Free radicals in Biology. New York, Academy Press, 1976; 1: 1- 43.

12. Halliwell B, Gutteridge J. Free radicals in Biology and Medicine. Tercera edición. Oxford, New York, 2000.

13. Halliwell B. Free radical, antioxidants and human disease, curiosity, cause, or consequence?. Lancet 1994; 344: 721 - 724.

14. Cheeseman K, Slater T. An introduction to free radical biochemistry. In: Cheeseman K, Slater T, editors. Free radicals in medicine. London (UK): Churchill Livingstone, 1993; 481 493.

15. Halliwell B, Chirico S. Lipid peroxidation: its mechanism, measurement and significance. Am J Clin Nutr 1993; 57: 715 -725 .

16. Halliwell B, Gutteridge J, Cross C. Free radicals, antioxidants, and human disease: Where are we now? J Lab Clin Med 1992; 119: $598-620$.

17. Lorenzi R. Efeito do Glicolaldeído sobre parámetros de estresse oxidativo no rim, fígado e coração de ratos Wistar. [Tesis de maestría] Porto Alegre (Brasil). Universidad Federal do Rio Grande do Sul. Programa de Pos graduación en Ciencias Biológicas. 2010.

18. Halliwell B. Oxidants and human disease: some new concepts. FASEB J 1987: 358 - 364. 
19. Morrisey P, O 'Brien N. Dietary antioxidants in health and disease. Int Dairy J. 1998; 8: 463 - 472.

20. Kehrer J. Free radicals as mediators of tissue injury and disease. Crit rev Toxicol. 1993; 23: $21-48$.

21. Llesuy S. Introducción y especies activas de oxigeno. En: estresse oxidativo e antioxidantes. Argentina. Editora: ULBRA. 2002: $22-32$.

22. Kolb H. Química para el nuevo milenio. Editora Pearson Hall Hispanoamérica. Octava edición. México. 1999

23. Spirito G, Cannellorro M, Breitbart G. Introducción a la medicina hiperbárica y metabolismo del oxígeno. Revitalair [en línea] 2011 [accesado 10 de octubre del 2012]. Disponible en: http://www.sambyh.com/v2/doc/Fisiologia-del-Oxigeno.pdf

24. Cartwright J. Del flogisto al oxígeno estudio de un curso práctico en la revolución química, materiales de historia de la ciencia. [en línea]. 2000. [fecha de acceso 30 de agosto del 2012]. Disponible en: http://www.gobcan.es/educacion/3/Usrn/fundoro/archivos $\% 20$ adjuntos/publicaciones $/ \mathrm{mhc} / \mathrm{fl}$ web cap/ flogisto.pdf.

25. Bertomeu J, García A. La revolución química entre la historia y la memoria. Universidad de Valencia. 2006.

26. Campbell M. Bioquímica. 2000; Porto Alegre: Artmed. 753.

27. Boveris A, Chance B. The mitochondrial generation of hydrogen peroxide. Biochem J. 1973; 134: 707-716.

28. Pourova J, Kottova M, Voprsalova M, et al. Reactive oxygen and nitrogen species in normal physiological processes. Acta physiol. 2010; 198: 15-35.

29. Perón J, López J, López T. Radicales libres en la biomedicina y estrés oxidativo. Rev. Cubana Med. Milit. 2001; 30 (1): 15 -20 .

30. Barb J, Araujo A, Fernandes T, et al. Myocardial antioxidant and oxidative stress changes due to sex hormones. Braz J Med Biol Res. 2002; 35: 1075 - 1081.

31. Morgan - Martins M. Reposiçao de estrogênio Dimui o daño oxidativo, aumenta a atividade das enzimas antioxidantes e melhora a funçao cardiaca em ratas. PPG em ciencias biológicas. Fisiología. Volume Tese de Doutorados. Porto Alegre (Brasil). UFRGS, 2003.

32. Oga Z. Fundamentos de toxicología. Segunda edición. Editora Atheneu, São Paulo. 2003: 39- 44.

33. Green M, Hill H. Methods en Enzymol, 1984; 105: 3 - 9.

34. Yu P. Cellular defenses against damage from reactive oxygen species. Physiological Reviews. 1994; 74 (1): 139 - 162.

35. Meneghini R. A toxicidade do oxigênio. Ciência Hoje. 1987: $57-62$.

36. Lobo V, Patil A, Phatak A, et al. Free radicals, antioxidants and functional foods: Impact on human health. Pharmacogn Rev. 2010; 4(8).

37. Augusto O. Radicais libres: bons, maus e naturais: Sao Paulo: oficina de textos. Sao Paulo (Brasil). 2006

38. Elahi M, Kong Y, Matata B. Oxidative stress as a mediator of cardiovascular disease. Oxidative and cellular Longevity. Australia; noviembre - diciembre 2009.
39. Gutierrez J. Daño oxidativo, radicales libres y antioxidantes. Rev Cubana Med Militar. Cuba; 2002; 31 (2): 126 - 33.

40. Halliwell B. Reactive species and antioxidants redox biology is a fundamental theme of aerobic life. Plant Physiol 2006; 141: $312-22$.

41. Punchard N, Kelly F. free radicals: a practical approach. Oxford University Press, 1996

42. Pincemail J, Free radicals and antioxidants in human diseases. En: Analysis of free radicals in biological systems, Favier et al. 1995.

43. Boveris A. Biochemistry of free radicals: from electrons to tissues. Buenos Aires (Argentina). 1998; 58: 350- 356.

44. Días J, Serramo E, Acosta F, et al. References intervals for four biochemistry analyttes in plasma for evaluating oxidative stress and lipid peroxidation in human plasma. Clin Chem. 1998; 44(10): $2215-2217$

45. Nichols D, Fergunson J. Bioenergetics 3. Cuarta edición. Editora Academic Express, San Diego. 2001: 127 - 129.

46. Mandavilli B, Van Houten B. Mitochondrial DNA repair and aging. Mutatio Research. 2002; $509(1-2): 127$ - 132.

47. Fridovich I. Oxygen toxicity: a radical explanation. J. Exp. Biol. 1998, 201: 1-15.

48. Felippe J, Percário S. Radicais libres em medicina intensiva. Rev. Bras. Terap. Intens. 1991; 3(3): 66 - 72.

49. Garcez M, Bordin D, Peres W, et al. Radicais livres e espécies reativas. En: radicais libres a resposta celular ao estresse oxidativo. Editora da ULBRA. Canoas- RS (Brasil). 2004; 13 - 31.

50. Aruoma O. nutrition and health aspects of free radicals and antioxidants. Food Chem Tox. 1994; 32 (7): 671 - 683.

51. Cadenas E. Biochemistry of oxygen toxicity. Annu. Rev Biochem. 1989; 58: 79-110.

52. Epe B. Genotoxicity of singlet oxygen. Chem Biol Interact. 1991; 80: $239-260$.

53. Bradley D, Min D. Singlet oxygen oxidation in foods. Rev Food Sci Nutri. 1992; 32 (3): 211 - 236.

54. Beckman K, Ames B. The free theory of aging matures. Physiol Rev. 1998; 78 (2): 547- 581.

55. Morgan M. Estresse oxidative e antioxidantes. En: Radicais libres no proceso saúde - doença da bancada a clínica. Curitiba- PR (Brasil). 2012.

56. Profert.com.br. Diagnóstico. Programa de Reprodução Assistida [en línea]. São Paulo (Brasil). 2011. [Acceso: 12 de octubre del 2012 ]. Disponible en: www.profert.com.br/diagnost061. htm

57. Giulivi C, Poderoso J, Boveris A. Production of nitric oxide by mitochondria. J. Biol. Chem. 1998; 273: 11038 - 11043.

58. Poderoso J, Carreras M, Lisdero C, et al. Nitrix oxide inhibitis electron transfer and increases superoxide radical production in rat heart mitocondria and submitochondrial particles. Arch Biochem Biophys. 1996; 328: 85 - 92.

59. Radi R, Cassina A, Hodara R, et al. Peroxynitrite reactions and formation in mitocondria. Free Radic Biol Med. 2002; 33: $1451-1464$. 
60. Ghafourifar P, Schenk U, Klein S. Mitochondrial nitric oxide synthase stimulation causes cytochrome $\mathrm{c}$ release from isolated mitochondria. J. Biol. Chem. 1999; 274: 31185 - 31188.

61. Grajales O. Apuntes de bioquímica vegetal. Bases para su aplicación fisiológica. Cuautitlán (México). 2005: 23- 24.

62. Delvin T. Bioquímic: libro de texto con aplicaciones clínicas. Editorial Reverté S. A. Barcelona (España). 2006: 476- 477.

63. Peres W. Radicais libres em niveis biológicos. Editorial Educar. 1995: $11-43$

64. Slater T. Free radical mechanism in tissue injury. Biochem J. 1984; 222: 1 - 15 .

65. Ugartondo V. Caracterización de derivados polifenólicos obtenidos de fuentes naturales. Citotoxicidad y capacidad antioxidante frente a estrés oxidativo en modelos celulares. Universitat de Barcelona. Facultad de farmacia. 2009.

66. HalliwellB. Antioxidants: The Basis - What they are and how to evaluate them. En: Antioxidants in deeseases: Mecanisms and therapy. SIES. Academic Press. California. 1997.

67. Chaudoére J, Ferrari R. Intracellular antioxidants from chemical biochemical mechanisms. Food Chem Toxicol. 1999; 37: $949-962$.

68. Bandyopadhyay U. Das D, Banerjee R. Reactive oxygen species: oxidative damage and pathogenesis. Curr Sci. 1999; 77: $658-666$
69. Borella M, Varela Q. Antioxidantes enzimáticos. En: Radicais libres e a resposata celular ao estresse oxidativo. Editora da ULBRA. Canoas- RS (Brasil). 2004: 35 - 49.

70. Zelko I, Mariani T, Foiz R. Superoxide dismutase multigene family: a comparision of the $\mathrm{Cu}-\mathrm{Zn}-\mathrm{SOD}$ (SOD1), MnSod (SOD2) and EC-Sod (SOD3) gene structures and expression. Free Radic. Biol. Med. 2002; 33 (3): 337 - 349.

71. Power S, Lennon S. Analysis of cellular responses to free radicals: focus on exercise and skeletal muscle. Proc Nutr Soc. 1999; 58: 1025 - 1033

72. Arthur J. The glutathione peroxidases. Cell Mol Life Sci. 2000; 57: $1825-1835$.

73. Allan C, Lacourciere G, Stadtman T. responsiveness of selenoproteins to dietary selenium. Ann Rev Nutr. 1999; 19: 1 - 16.

74. Holben D, Smithe A. The diverse role of selenium within selenoproteins: a review. J Am Diet Assoc. 1999; 99: 836 - 843.

75. Larkins N. Free radical biology and pathology. J Equine Vet Sci. 1999; 19: 84- 89.

76. Olinescu R, Kummerow F. Fibirnogen is an efficient antioxidant. J Nutr Biochem. 2001; 12: 162- 169.

77. Burns R. Fundamentos de química. Editorial Pearson Educación. 2003.

78. Dröge W. Free radicals in the physiological control of cell function. Physiol. Rev. 2002; 82: 47 - 95. 